\title{
Mejorar la competencia motriz no predice un cambio en la autopercepción en adultos
}

\author{
Improvement in motor competence does not predict changes in self- \\ perception in adults
}

\author{
* Judith Jiménez-Díaz, **María Morera-Castro, *,** Gerardo Araya-Vargas
}

Jiménez-Díaz, J., Morera-Castro, M., \& Araya-Vargas, G. (2020). Mejorar la competencia motriz no predice un cambio en la autopercepción en adultos. Revista Ciencias de la Actividad Física UCM, No 21(2), julio-diciembre, 1-13. DOI: http://doi.org/10.29035/rcaf.21.2.7

\section{RESUMEN}

Objetivo. El objetivo del presente estudio fue determinar si mejorar el desempeño motor, predice la autopercepción en adultos. Métodos: Un total de 55 personas ( $M=20 \pm 2$ años) fueron asignadas aleatoriamente a uno de tres grupos: práctica en bloque, práctica aleatoria y grupo control. Para evaluar la autopercepción se utilizó el Perfil de Autopercepción para Adultos, en los dominios de habilidades deportivas y apariencia física. El Instrumento para la Evaluación de los Patrones Básicos de Movimiento en Adultos, se utilizó para evaluar el desempeño motor, en las destrezas básicas de saltar y lanzar. Se aplicó una intervención motriz de siete sesiones, utilizando práctica en bloque y aleatoria. Resultados: Los resultados determinaron que los grupos que realizaron práctica en bloque o aleatoria mejoraron significativamente el desempeño en saltar y lanzar, y fueron superiores al grupo control en el post test. El análisis de regresión múltiple indicó que el cambio en el desempeño, el sexo o el tipo de práctica realizada no predicen significativamente el cambio en la competencia percibida en habilidades deportivas $\left(R^{2}=.038 ; p=.739\right)$, ni en la autopercepción de la apariencia física $\left(R^{2}=.008 ; p=.366\right)$. Conclusión: En conclusión, la práctica en bloque y práctica aleatoria mejoraron el desempeño en los patrones, pero esta mejoría no predice cambios en la autopercepción de esta muestra.

Palabras clave: desempeño motor, competencia motriz, competencia percibida, salud.

* Escuela de Educación Física y Deportes, Universidad de Costa Rica, San José, Costa Rica.

** Escuela de Ciencias del Movimiento Humano y Calidad de Vida, Universidad Nacional, Heredia, Costa Rica. 


\section{ABSTRACT}

Purpose: The aim of the present study was to determine whether improving motor performance predicts selfperception in adults. Methods: A total of 55 participants ( $M=20 \pm 2$ years old) were randomly assigned to one of three groups: blocked practice, random practice, and the control group. To evaluate self-perception, The SelfPerception Profile for Adults was used in the sports skills and physical appearance domains. The Test of Fundamental Motor Skills in Adults was used to evaluate motor performance in jumping and throwing skills. A seven-session motor intervention was applied, using blocked and random practice. Results: The results showed that the groups that performed blocked or random practice significantly improved their jump and throw performances and were superior to the control group in the post-test. Multiple regression analysis indicated that the improvement in motor performance, sex or type of practice performed did not significantly predict a change in perceived competence in sports skills $\left(R^{2}=.038 ; p=.739\right)$, nor in self-perception of physical appearance $\left(R^{2}=.008 ; p=.366\right)$. Conclusion: In conclusion, the blocked practice and random practice improved the performance in the motor skill, but this improvement does not predict changes in the self-perception of this sample.

Key words: motor development, motor competence, perceived competence, health.

\section{Introducción}

Estudios realizados en las últimas décadas, indican que la competencia motriz (CM) está asociada positivamente con el nivel de actividad física (Holfelder \& Schott, 2014), con las capacidades cardio-respiratorias, fuerza y resistencia muscular (Cattuzzo et al., 2016, Lubans, Morgan, Cliff, Barnett \& Okely, 2010), con la autopercepción (Barnett, Ridgers, Zask \& Salmon., 2015, LeGear et al., 2012); además, está inversamente relacionado al peso corporal (Cattuzzo et al., 2016), en la población infantil y adolescente.

La CM es el desempeño actual que presenta la persona al ejecutar diferentes destrezas, las cuales implican la coordinación y control del movimiento humano (Cattuzzo et al., 2016). Estas destrezas incluyen el desempeño en patrones básicos de movimiento (PBM), los cuales son destrezas motrices simples consideradas fundamentales para la ejecución de destrezas más complejas, como las destrezas específicas en los deportes (Gabbard, 2018, GaIlahue \& Ozmun, 2005, Logan et al., 2012, Morgan et al., 2013, Van Capelle, Broderick, van Doorn, Ward \& Parmenter, 2017). Comúnmente los PBM se clasifican en movimientos locomotores (saltar a distancia, correr, galopar, entre otras) y manipulativos (lanzar por encima del hombro, batear, patear, entre otras)
(Gabbard, 2018, Gallahue \& Ozmun, 2005, Logan, Robinson, Wilson \& Lucas, 2012).

Se ha demostrado que un desempeño eficiente de los PBM no se adquiere de manera natural, requiere de práctica y de equipamiento apropiado para la edad y el tamaño corporal (Logan, Robinson, Wilson \& Lucas, 2012). Los estudios recientes indican que el desempeño en PBM se puede mejorar significativamente por medio de intervenciones motrices, en personas de diferentes edades (Chaves, Jiménez \& Salazar, 2018, Logan et al., 2012, Morgan et al., 2013, Van Capelle, Broderick, van Doorn, Ward \& Parmenter, 2017, Wick et al., 2017). No obstante, se ha encontrado que un gran porcentaje de adolescentes y adultos no presentan un desempeño eficiente en estas destrezas (Jiménez, Salazar \& Morera, 2015, Morgan et al., 2013, O'Brien, Belton \& Issartel, 2016).

Por otro lado, la autopercepción (también conocida como autoconcepto) es un constructo multidimensional, que representa la interpretación que cada persona tiene de sí mismo en distintas características personales (Fu, Gao, Hannon, Shultz, Newton \& Sibthorp, 2013, Gabbard, 2018, Gentil, Ortega, Sánchez, Ruz \& Lara, 2019) que incluye la autopercepción de la apariencia física, la competencia 
Jiménez-Díaz, J., Morera-Castro, M., \& Araya-Vargas, G. (2020). Mejorar la competencia motriz no predice un cambio en la autopercepción en adultos. Revista Ciencias de la Actividad Física UCM, №21(2), julio-diciembre, 1-13. DOI: http://doi.org/10.29035/rcaf.21.2.7

motriz percibida, la aceptación social, la competencia en las habilidades deportivas, entre otras características (Messer \& Harter, 2012). A su vez, la autopercepción está relacionada con la CM (Famelia, Tsuda, Bakhtiar \& Goodway, 2018, Robinson et al., 2015, True, Brian, Goodway \& Stodden, 2017), con el nivel de actividad física (Babic, Morgan, Plotnikoff, Lonsdale, White \& Lubans, 2014) y con las capacidades físicas relacionadas a la salud (Haugen, Ommundsen \& Seiler, 2013). En diferentes estudios se ha encontrado un cambio positivo en la autopercepción (de las habilidades físicas, de la apariencia física, entre otras) después de diversas sesiones de programas físicos o intervenciones motrices (Castillo, Corvalán, Sazo \& Concha, 2017, Fu et al., 2013, Lubans, Aguiar \& Callister, 2010, Morano, Colella, Rutigliano, Fiore, Pettoello \& Campanozzi, 2014, Opdenacker, Delecluse \& Boen, 2009), sin embargo, este efecto no siempre se presenta (Calderón, Martínez \& Martínez, 2013, Lindwall \& Lindgren, 2005, Morgan, Saunders \& Lubans, 2012).

La relación entre la CM, la autopercepción, el nivel de actividad física y las capacidades físicas relacionadas con la salud, en la niñez y adolescencia, se ha analizado por medio de un modelo explicativo, donde se establece una relación directa entre la CM y la actividad física; entre la autopercepción y la actividad física; entre la autopercepción y CM; entre las capacidades físicas relacionadas con la salud y la CM; y entre capacidades físicas relacionadas con la salud y la autopercepción, y su implicación en el riesgo de obesidad (Robinson et al., 2015, Stodden et al., 2008). Además, se considera la CM como un predictor de estos factores (Barnett et al., 2016). Sin embargo, tomando en cuenta la baja prevalencia de personas adultas con un desempeño eficiente en los PBM, en el presente estudio se plantearon dos objetivos: primero, establecer el efecto de dos tipos de práctica (bloque y aleatoria) sobre el desempeño de los PBM (saltar a distancia y lanzar por encima del hombro) y la autopercepción de la apariencia física (AF) y la competencia percibida de las habilidades deportivas (HD) y en personas adultas. Segundo, determinar si el cambio en la CM, el sexo o el tipo de práctica realizada predice el cambio en la AF y la HD de las personas adultas.

\section{METODOLOGÍA}

\section{Diseño}

El presente estudio muestra un enfoque de investigación cuantitativo. En la primera etapa, se cuenta con un diseño experimental univariado, donde se contó con asignación aleatoria a los grupos de investigación, mediciones pretest y postest, dos grupos experimentales, además de un grupo control. En la segunda etapa, el diseño es de tipo multivariado predictivo, donde se busca predecir el cambio en dos variables dependientes, a partir de un grupo de variables independientes (Hair, Anderson, Tatham \& Black, 1999). La muestra utilizada fue seleccionada por conveniencia, a estudiantes matriculados en una universidad en Costa Rica.

\section{Participantes}

En el presente estudio participaron de forma voluntaria 55 estudiantes de una universidad en Costa Rica, con edades entre los 18 y 26 años. En la Tabla 1 se muestran las características descriptivas de la muestra de estudio. Los criterios de elegibilidad para participar en el estudio fueron: ser estudiante activo de la universidad, estar matriculado en un curso de actividades físicas o deportivas impartido en la universidad. Mientras que los criterios de exclusión incluyeron presentar una dificultad motriz y/o de aprendizaje, o ser estudiante activo de la carrera de Ciencias del Movimiento Humano o Educación Física. Todas personas participantes firmaron el consentimiento informado. 
Tabla 7

Características de los participantes por sexo..

\begin{tabular}{lcccccc}
\hline & \multicolumn{2}{c}{$\begin{array}{c}\text { Masculino } \\
\text { Características }\end{array}$} & \multicolumn{2}{c}{$\begin{array}{c}\text { Femenino } \\
(n=15)\end{array}$} & \multicolumn{2}{c}{$\begin{array}{c}\text { Total } \\
(n=55)\end{array}$} \\
\cline { 2 - 7 } & $M$ & $D E$ & $M$ & $D E$ & $M$ & $D E$ \\
\hline Edad (años) & 20.35 & 2.15 & 19.88 & 1.58 & 20.27 & 2.00 \\
Talla $(\mathrm{m})$ & 1.72 & 0.07 & 1.58 & 0.06 & 1.68 & 0.09 \\
Peso (Kg) & 66.55 & 9.64 & 56.69 & 8.93 & 63.67 & 10.40 \\
IMC & 22.50 & 2.93 & 22.73 & 4.29 & 22.57 & 3.35 \\
\hline
\end{tabular}

Nota: $M=$ media, $D E=$ desviación estándar.

\section{Instrumentos}

Para evaluar la autopercepción se utilizó el instrumento de auto-reporte "Perfil de Autopercepción para Adultos" (Messer \& Harter, 2012), traducido y validado para su aplicación en población costarricense (Jiménez, Morera \& Araya, 2018). Este instrumento evalúa la autopercepción en 11 dominios específicos y un dominio de autopercepción global (para un total de 12 dominios). Para el presente estudio se utilizó el dominio de HD y AF, por presentar mayor relación conceptual con la CM, ambos con confiabilidad aceptables $(R=0.814$ y $R=0.855$, respectivamente) (Jiménez et al., 2018). Cada dominio se evalúa con un puntaje entre 1 y 4 puntos (Messer \& Harter, 2012); un puntaje cercano a 1 indica que la persona se percibe menos competente, mientras que un puntaje cercano a 4 indica que la persona percibe más competente, en ese criterio específico.

Para la evaluación del desempeño se utilizó el Instrumento para la Evaluación de los Patrones Básicos de Movimiento (IPBM). El IPBM presenta una validez de juicio de expertos, confiabilidad $(R=.92)$ y objetividad ( $R=$.86) aceptables (Jiménez, Salazar, \& Morera, 2013). Este instrumento evalúa el proceso de desempeño de 10 patrones básicos de movimiento, cinco locomotores (LM) y cinco de manipulación de objetos (MO). Cada movimiento se evalúa por medio de seis características observables, que en conjunto determinan un desempeño eficiente. Se puntúa un
"7" si se presenta el criterio y un "O" si no lo presenta. El puntaje de cada movimiento es de 0 a 12 puntos (en dos intentos), la subescala de LM es el promedio de los cinco patrones locomotores (correr, galopar, deslizar, saltar y brincar) y la subescala de $\mathrm{MO}$ es el promedio de los cinco patrones manipulativos (rebotar, lanzar, apañar, patear y batear), el desempeño total es el promedio de los 10 movimientos. Un puntaje mayor o igual a 9, implica que el desempeño es eficiente. En el presente estudio se utilizó únicamente la escala de movimiento de saltar a distancia y lanzar por encima del hombro. Se seleccionaron estos dos patrones por ser los que presentan menor desempeño en la población adulta en estudios previos (Jiménez \& Morera, 2016, Jiménez, Salazar \& Morera, 2015)

Para fines descriptivos de la muestra la talla y el peso se obtuvieron con una báscula de bioimpedancia (BF-682W TANITA) y un tallímetro portátil (Seca-213), respectivamente.

\section{Procedimientos}

Los procedimientos del presente estudio se apegaron a la declaración de Helsinki. Primeramente, se solicitaron los permisos antes las autoridades correspondientes de la universidad. Una vez, obtenidos los permisos, se efectuaron un total de 9 sesiones, en las cuales, en las sesiones 1 y 9 se realizaron las mediciones pre y post y en las sesiones 2 a 8 se 
aplicó el tratamiento (7 sesiones práctica). A continuación, se detalla cada sesión:

Sesión 1: se realizaron las mediciones pretest. Se firmó el consentimiento informado, se recolectó información personal (por medio de un cuestionario construido para dicho fin donde se indica edad, sexo, carrera en la cual se encuentra empadronado, entre otros); se obtuvo el peso y talla (una vez para fines descriptivos), y posteriormente se contestó el cuestionario de autopercepción, para las escalas de HD y AF. El índice de masa corporal (IMC) se obtuvo con la fórmula, $\mathrm{kg} / \mathrm{m}^{2}$. Por último, se evalúo el desempeño de los patrones saltar y lanzar.

Sesiones 2 a 8: se aplicaron las sesiones experimentales. Los participantes fueron asignados aleatoriamente a uno de tres grupos de investigación (Grupo A, B, C). La asignación de los participantes a los grupos experimentales y control, se buscó un volumen equitativo de género, fue a través del método de aleatorización simple, posteriormente cada grupo fue asignado nuevamente de manera aleatoria a uno de los tres tratamientos. Grupo A: en cada sesión de trabajo, los participantes de este grupo realizaron práctica aleatoria (PA) para promover el cambio en el desempeño motor en saltar y lanzar, la práctica realizada consistió en 20 intentos de cada destreza (20 saltos, 20 lanzamientos) organizados de forma aleatoria. En cada sesión, los participantes recibieron un papel con el orden de los intentos, los cuales eran diferentes en cada persona y en cada sesión. Grupo B: realizó práctica en bloque (PB) para promover el cambio en el desempeño de saltar y lanzar, en cada sesión la práctica consistió en 20 intentos de un patrón seguidos de 20 intentos del otro patrón. En cada sesión los participantes recibieron un papel indicando de cual destreza debían hacer los primeros 20 intentos y de cual destreza los segundos 20 intentos, por lo que en cada sesión el orden de trabajo podía ser variado. Grupo C: este grupo no realizó práctica específica de cada destreza, realizaron juegos predeportivos de fútbol, clases de acondicionamiento físico y clases de danza aeróbica (CG). En general cada sesión tuvo una duración de 30 minutos.

Sesión 9: se realizaron las mediciones postest. Se contestó el cuestionario de autopercepción, para las escalas de HD y AF; y se evalúo el desempeño de los patrones saltar y lanzar.

Todas las sesiones se llevaron a cabo en un gimnasio multiuso deportivo de la universidad; las evaluaciones y las sesiones de los grupos experimentales de PB y PA, fueron realizadas por el investigador principal, mientras que las sesiones del GC fueron realizadas por un profesor de Educación Física asignado por la Universidad, sin embargo, las actividades seleccionadas fueron planeadas en conjunto con el investigador principal.

\section{Análisis Estadístico}

Se calculó promedio y desviación estándar de las características de interés para describir la muestra. Se aplicó un análisis de varianza (ANOVA) de dos vías mixto (grupo [3] x medición [2]) con medidas repetida en el último factor, para las variables de autopercepción y desempeño motor. Se calculó el cambio (medición postest menos medición pretest) en el desempeño motor para cada patrón y el cambio en la autopercepción de las HD y AF. Por último, se aplicó la técnica de regresión múltiple (método de introducción) para determinar si el cambio en desempeño motor, el sexo o el tipo de práctica realizada predice el cambio en autopercepción. Se estableció un nivel de significancia de $\mathrm{p} \leq 0.05$. Se utilizó el programa de IBM-SPSS ${ }^{\circledR}$ versión 24 (IBM Corporación, New York, USA)

\section{Resultados}

Al aplicar un ANOVA de una vía para grupos independientes, no se encontró diferencias significativas 
Jiménez-Díaz, J., Morera-Castro, M., \& Araya-Vargas, G. (2020). Mejorar la competencia motriz no predice un cambio en la autopercepción en adultos. Revista Ciencias de la Actividad Física UCM, №21(2), julio-diciembre, 1-13. DOI: http://doi.org/10.29035/rcaf.21.2.7

entre los grupos al inicio del estudio en la variable de lanzar y saltar ( $p>$.05), ni en ninguno de los dos dominios de autopercepción ( $p$ > .05). En la Tabla 2 se muestran los resultados descriptivos para los patrones de lanzar y saltar, y el dominio de autopercepción de las HD y AF.

Tabla 2

Desempeño motor y autopercepción por grupo en cada medición.

\begin{tabular}{lcccccc}
\hline \multirow{2}{*}{ Variable } & \multicolumn{6}{c}{ Grupos } \\
\cline { 2 - 7 } & \multicolumn{2}{c}{$\begin{array}{c}\text { Bloque }(\mathrm{PB}) \\
(\mathrm{n}=19)\end{array}$} & \multicolumn{2}{c}{$\begin{array}{c}\text { Aleatorio }(\mathrm{PA}) \\
(\mathrm{n}=18)\end{array}$} & \multicolumn{2}{c}{$\begin{array}{c}\text { Control }(\mathrm{CC}) \\
(\mathrm{n}=18)\end{array}$} \\
\cline { 2 - 7 } & Pretest & Postest & Pretest & Postest & Pretest & Postest \\
\hline Lanzar & $5.89 \pm 2.99$ & $10.11 \pm 1.66$ & $5.73 \pm 2.94$ & $9.84 \pm 1.98$ & $5.52 \pm 2.69$ & $6.53 \pm 2.55$ \\
Saltar & $6.26 \pm 1.79$ & $10.11 \pm 1.91$ & $6.78 \pm 2.55$ & $10.05 \pm 1.61$ & $7.00 \pm 2.76$ & $8.05 \pm 2.30$ \\
Habilidades deportivas & $2.07 \pm 0.56$ & $2.04 \pm 0.52$ & $2.42 \pm 0.88$ & $2.67 \pm 0.86$ & $2.07 \pm 0.56$ & $2.14 \pm 0.65$ \\
Apariencia física & $2.42 \pm 0.52$ & $2.72 \pm 0.61$ & $2.80 \pm 0.85$ & $3.12 \pm 0.61$ & $2.49 \pm 0.84$ & $2.70 \pm 0.58$ \\
\hline
\end{tabular}

Nota: datos se presenta $M \pm D E . M=$ media, $D E=$ desviación estándar

Al aplicar un ANOVA de 2 vías mixto (grupo [3] $x$ medición [2]) con medidas repetida en el último factor, para las variables de autopercepción de HD no se encontró interacción significativa entre gruposy medición ( $F=0.906 ; p=.417$ ), ni para los efectos principales de medición ( $F=1.757 ; p=.191)$. Sin embargo, si se encontró una diferencia significativa en el factor de grupos ( $F=5.304 ; p=.008)$, siendo el grupo de práctica aleatoria significativamente diferente del control y el grupo de práctica en bloque. El PA se percibió más competente que los PB y GC.

En la variable de AF no se encontró interacción significativa entre grupos y medición $(F=0.212 ; p=$ .810), ni para los efectos principales de grupo ( $F=$ 1.920; $p=$.157). Sin embargo, si se encontró una diferencia significativa en el factor de medición ( $F=$ 10.514; $p=.002$ ), el puntaje de la medición postest fue significativamente mayor que la medición pretest.

En la variable de desempeño en el patrón de lanzar, se encontró una interacción significativa entre grupos y medición ( $F=6.633 ; p=.003)$. Además, se encontró resultados significativos en los efectos principales del factor de grupo $(F=3.926 ; p=.026) \mathrm{y}$ medición ( $F=61.179 ; p<.001$ ). El análisis pos-hoc de la interacción indicó que los grupos de práctica en bloque y aleatorio mejoraron el desempeño significativamente, y en la medición postest ambos grupos presentan un mejor desempeño que el grupo control.

En la variable de desempeño en el patrón de saltar, se encontró una interacción significativa entre grupos y medición ( $F=7.859 ; p=.001$ ). Además, se encontró resultados significativos en los efectos principales del factor de medición ( $F=80.684$; $p<$ .001). Sin embargo, no se encontró resultados significativos para el factor de grupo ( $F=0.882 ; p=.420)$. El análisis pos-hoc de la interacción indicó que los grupos de práctica en bloque y aleatorio mejoraron el desempeño significativamente, y en la medición post test ambos grupos presentan un mejor desempeño que el grupo control.

Se analizó el modelo donde el cambio en saltar, lanzar, el sexo y el grupo de práctica predicen el cambio en autopercepción en HD y AF. El modelo para predecir el cambio en las HD no fue significativo $\left(R^{2}=.038 ; p=.739\right)$. El cambio en desempeño de $\operatorname{saltar}(B=.03 ; t=0.964 ; p=.340)$ y lanzar $(B=-.009 ; t$ $=-0.287 ; p=.775)$, el sexo $(B=.131 ; t=0.661 ; p=.511)$, ni el tipo de práctica realizada $(B=.105 ; t=0.828 ; p=$ 411) son predictores significativos.

El modelo para predecir la AF tampoco fue significativo ( $\left.R^{2}=.008 ; p=.366\right)$; el cambio en desempeño de saltar $(B=.03 ; t=0.753 ; p=.455)$ y lanzar $(B$ $=-.008 ; t=-0.255 ; p=.800)$, el $\operatorname{sexo}(B=.364 ; t=1.817$; 
$p=.076)$, ni el tipo de práctica realizada $(B=-.02 ; t=$ $-0.227 ; p=.821)$ son predictores significativos.

\section{Discusión}

La relación entre la CM y la competencia percibida ha sido estudiada en la población infantil y adolescente (Robinson et al., 2015), sin embargo, pocos estudios han examinado el impacto de una intervención motriz en la autopercepción de la AF, la competencia percibida en HD y la CM; o la relación entre ellas en la población de personas adultas. En este estudio se plantearon dos objetivos, uno en términos de causa-efecto y otro en términos de predicción. Con respecto al primer objetivo se encontró que ambos tipos de práctica (PA y PB) mejoran el desempeño en los patrones de saltar y lanzar en personas adultas. Además, los resultados sugieren que realizar ambos tipos de prácticas enfocadas en la CM y actividades físicas (por ejemplo, juegos predeportivos de fútbol, clases de acondicionamiento físico y clases de danza aeróbica) favorecen positivamente el cambio en la autopercepción de la AF, pero no en la competencia percibida de las HD. Sin embargo, en el segundo objetivo se encontró que la mejoría en el desempeño de saltar y lanzar, el sexo y el tipo de práctica realizada no son predictores de la autopercepción de la AF ni de la competencia percibida de las HD.

En relación con el primer objetivo, los resultados de la presente investigación motriz son apoyados por diversos estudios, donde se ha encontrado que una intervención motriz planificada acorde a las características de las personas participantes mejora la CM en diversas poblaciones (Chaves et al., 2018, Logan et al., 2012, Morgan et al., 2013). La práctica en bloque y aleatoria fue planificada acorde a las necesidades y objetivos de las personas adultas, ya que este tipo de práctica ha demostrado que es efectiva para el aprendizaje de destrezas motrices (Jiménez, Salazar \& Morera, 2015), por lo que era de esperarse una mejoría en las destrezas. No obstante, cabe resaltar que la práctica de actividad física sin que el fin último fuese el mejoramiento de las destrezas, como lo realizado en el GC, no es efectiva para mejorar la CM. Este hallazgo va en concordancia con los resultados de otros estudios, donde el grupo de comparación -que realiza juego libre u otro tipo de actividad física- no mejoró el desempeño destrezas motrices (Logan et al., 2012; Piek et al., 2013). Los resultados del presente estudio refuerzan que el desempeño motor en PBM requiere de actividades diseñadas acorde a las necesidades de las personas participantes, y se debe tomar en cuenta que no se desarrollan de manera natural, por lo que, para lograr un desempeño eficiente de los PBM se deben enseñar, practicar y reforzar acorde a las características de las personas (Chaves et al., 2018, Logan et al., 2012, Van Capelle, Broderick, van Doorn, Ward \& Parmenter, 2017, Wick et al., 2017). Una posible explicación se debe a la oportunidad que experimentan las personas que participan de actividades planificadas con el objetivo de mejorar una destreza específica, en este caso el patrón de saltar y lanzar, con una instrucción acorde a sus necesidades, con una cantidad de sesiones suficiente, brinda las condiciones necesarias para que las personas mejoren su desempeño motriz (Goodway, Crowe \& Ward, 2003, Logan et al., 2012).

Adicional a lo anterior, los resultados del presente estudio sugieren que todas las actividades realizadas: PB, PA y GC (juegos predeportivos de fútbol, clases de acondicionamiento físico y clases de danza aeróbica) favorecen un cambio positivo en la autopercepción de la AF, pero no en la competencia percibida de las HD. En investigaciones previas se ha encontrado que realizar diversos tipos de actividad física favorece la autopercepción en diferentes dominios en poblaciones infantiles y adolescentes (Fu et al., 2013, Lubans, Aguiar \& Callister, 2010, Morano et al., 2014). Específicamente, en la autopercepción de la AF, estudios similares al presente estudio encontraron que, al realizar un programa de entrenamiento contra resistencia, mejoró la autopercepción de la AF en mujeres adolescentes (Lubans, Aguiar \& Callister, 2010), a su vez en un grupo de adultos mayores, se encontró que un programa estructurado de ejercicio proporcionó beneficios en la 
percepción de la condición física, deportiva y el atractivo corporal (Opdenacker, Delecluse \& Boen, 2009). Por el contrario, los resultados del presente estudio difieren con los hallazgos de una investigación donde no se encontró un cambio significativo en la percepción de la AF en un grupo de adolescentes, después de un programa de prevención de obesidad (Morgan, Saunders \& Lubans, 2012). En relación con la competencia percibida de las HD, en el presente estudio, no se encontró que ninguna de las actividades realizadas influyera en las HD, lo cual es congruente con los resultados de un estudio previo, donde el entrenamiento contra-resistencia no influyó en la competencia deportiva de los hombres ni de las mujeres adolescentes (Lubans, Aguiar \& CaIlister, 2010). De manera similar, no se encontró efecto significativo de la intervención en la competencia deportiva percibida, después de que un grupo de adolescentes participaron en un programa de prevención de obesidad (Morgan, Saunders \& Lubans, 2012). Sin embargo, a diferencia del presente estudio, en un grupo de adultos mayores si se encontró diferencias en la competencia percibida de las HD (Opdenacker, Delecluse \& Boen, 2009). Los efectos de diversas actividades físicas en distintos dominios de la autopercepción son variados; por lo que los investigadores han planteado que existen muchos factores que influyen en los resultados, entre ellos se encuentran las escalas utilizadas para evaluar cada dominio de la autopercepción (Barnett, Ridgers \& Salmon, 2015), la edad de los participantes (Lubans, Aguiar \& Callister, 2010) y la duración y características de la intervención de actividad física realizada (Fu et al., 2013).

El segundo objetivo del presente estudio se planteó en términos de predicción, donde se encontró que la mejoría en el desempeño de la CM, el sexo y el tipo de práctica realizada no son predictores de la autopercepción de la AF ni en la competencia percibida de las HD. En estudios previos se encontró que el desempeño de PBM de control de objetos si es predictor de la competencia percibida en destrezas de control de objetos en un grupo de infantes (Barnett, Ridgers, \& Salmon, 2015). También se ha encontrado una relación positiva entre el nivel de actividad física y la autopercepción atlética y social (Stein, Fisher, Berkey \& Colditz, 2007). Pero, a diferencia de los estudios anteriores la edad de los participantes puede ser un factor que influye en el resultado del presente estudio. Ya que, aunque la relación entre autopercepción y CM está comprobada en etapa infantil y adolescente (Robinson et al., 2015), poco se sabe de esta relación en la etapa adulta (Wang, Liu \& Bian, 2013). Si bien, se ha planteado que se espera que esta elación sea similar en la etapa adulta (Stodden et al., 2008), el cambio en la CM no predice la autopercepción en la población del presente estudio. Una posible explicación de los resultados encontrados en este estudio se puede deber a la estabilidad cognitiva que pueden presentar las personas adultas, lo que disminuye las posibilidades de un cambio en poco tiempo. Esto se debe a que la autopercepción de las personas adultas es más similar a lo real, ya que se encuentran en un nivel de madurez cognitivo, contrario a la población infantil que tiende a sobreestimar su autopercepción en diferentes dominios (Barnett et al., 2018). Otra posible explicación, puede ser el tipo de escala utilizada, y que la congruencia entre lo que evalúa la escala de autopercepción y la destreza evaluada influyen en la relación entre estos conceptos (Barnett et al., 2018, Barnett, Ridgers, Zask, \& Salmon, 2015).

Una implicación de los resultados de este estudio es que se demuestra que se pueden mejorar los PBM a través de intervenciones cortas en población adulta joven, lo que es esencial para mantener niveles saludables de actividad física. A su vez, esta intervención permitió que la autopercepción de la AF también obtenga un cambio significativo. Tomando en cuenta la relación que existe entre el desempeño de los PBM, la autopercepción de las personas, el nivel de actividad física y las capacidades físicas relacionadas a la salud en la población infantil y adolescente (Barnett et al., 2018, Robinson et al., 2015, Stodden et al., 2008), es posible suponer que, si se logra un cambio en estas características, se podría esperar un impacto en el nivel de actividad física de las personas, así como en la participación en 
Jiménez-Díaz, J., Morera-Castro, M., \& Araya-Vargas, G. (2020). Mejorar la competencia motriz no predice un cambio en la autopercepción en adultos. Revista Ciencias de la Actividad Física UCM, №21(2), julio-diciembre, 1-13. DOI: http://doi.org/10.29035/rcaf.21.2.7

actividades deportivas/recreativas y por ende una mejoría en las capacidades físicas relacionadas a la salud, efecto del aumento del nivel de actividad física. Ya que una persona con mayor autopercepción estaría más motivada a participar en actividades físicas, lo que aumentaría tanto su nivel de actividad física, como su CM (Stodden et al., 2008). Por lo que, los profesionales en movimiento humano deben buscar estrategias, para mejorar no solo la CM de sus estudiantes, sino también fortalecer las autopercepciones en los diferentes dominios, ya que para aumentar los hábitos saludables y, por ende, la calidad de vida de las personas, se requiere de una mejor comprensión de su propio cuerpo y de sus autopercepciones (Cabral \& Leal, 2017).

No obstante, se sugiere mayor cantidad de investigaciones en esta área enfocada en la población adulta.

Sin embargo, cabe destacar que los resultados de este estudio no son generalizables a toda la población, pero si sugieren que, al menos para un grupo de adultos-jóvenes que comparten las características de la muestra, las intervenciones motrices presentan efecto en el desempeño motor y en la percepción de la AF. Además, es necesario resaltar que una limitación del estudio fue evaluar dos destrezas simples, en vez de las diez que evalúa la prueba; por lo que se recomienda realizar estudios donde se examine esta relación con otros patrones básicos de movimiento. Adicionalmente, para futuras investigaciones se sugiere el planteamiento de diferentes metodologías de investigación donde se incrementen las sesiones experimentales y los tipos de actividades físicas realizadas con el fin de explorar si la autopercepción de las HD puede ser mejorada. Asimismo, es importante seguir explorando cuales pueden llegar a ser los predictores de la competencia percibida en adultos jóvenes. A su vez, se recomienda ampliar la edad de la muestra de estudio, a una más representativa de la etapa adulta.

\section{Conclusiones}

Se concluyó que intervenciones motrices utilizando práctica en bloque o práctica aleatoria con una duración de siete semanas, que presentan actividades planeadas con el objetivo de mejorar una destreza motriz de PBM fue efectiva para mejor el desempeño en saltar y lanzar.

Además, se determinó que realizar diferentes tipos de actividades físicas como clases de danza aeróbica, juegos predeportivos de fútbol, clases de acondicionamiento físico y la intervención motriz enfocada en PBM mejoran la AF la de las personas adultas jóvenes. Sin embargo, ninguna de las actividades anteriores mejoró la HD.

En términos de la predicción realizada, se concluyó que el sexo, las prácticas efectuadas y el cambio en el desempeño de los dos PBM evaluados, no predicen el cambio en las habilidades deportivas ni en la apariencia física en personas adultas-jóvenes.

\section{REFERENCIAS BIBLIOGRÁFICAS}

Babic, M. J., Morgan, P. J., Plotnikoff, R. C., Lonsdale, C., White, R. L., \& Lubans, D. R. (2014). Physical activity and physical self-concept in youth: Systematic review and metaanalysis. Sports Medicine, 44(11), 1589-1601. DOI: 10.1007/s40279-014-0229-z

Barnett, L. M., Lai, S. K., Veldman, S. L., Hardy, L. L., Cliff, D. P., Morgan, P. J., ... Okely, A. (2016). Correlates of Gross Motor Competence in Children and Adolescents: A Systematic Review and Meta-Analysis. Sports Medicine, 46(11), 1663-1688. DOI: 10.1007/s40279-016-0495-z 
Jiménez-Díaz, J., Morera-Castro, M., \& Araya-Vargas, G. (2020). Mejorar la competencia motriz no predice un cambio en la autopercepción en adultos. Revista Ciencias de la Actividad Física UCM, № 21(2), julio-diciembre, 1-13. DOI: http://doi.org/10.29035/rcaf.21.2.7

Barnett, L. M., Lubans, D. R., Timperio, A., Salmon, J., \& Ridgers, N. D. (2018). What is the Contribution of Actual Motor Skill, Fitness, and Physical Activity to Children's SelfPerception of Motor Competence? Journal of Motor Learning and Development, 6(s2), S461-S473.

DOI: https://doi.org/10.1123/jmld.2016-0076

Barnett, L. M., Ridgers, N. D., \& Salmon, J. (2015). Associations between young children's perceived and actual ball skill competence and physical activity. Journal of Science \& Medicine in Sport, 18(2), 167-171. DOI: 10.1016/j.jsams.2014.03.001

Barnett, L. M., Ridgers, N. D., Zask, A., \& Salmon, J. (2015). Face validity and reliability of a pictorial instrument for assessing fundamental movement skill perceived competence in young children. Journal of Science and Medicine in Sport, 18(1), 98-102. DOI: 10.1016/j.jsams.2013.12.004

Cabral, M., \& Leal, A. (2017). Nivel de actividad física y la percepción de la imagen corporal de estudiantes-Una revisión sistemática. Ciencias De La Actividad Física UCM, 18(1), 61-72. Recuperado de http://revistacaf.ucm.cl/article/view/109

Calderón, A., Martínez de Ojeda, D., \& Martínez, I. M. (2013). Influencia de la habilidad física percibida sobre la actitud del alumnado tras una unidad didáctica basada en Educación Deportiva (Influence of perceived skill on the attitude of students after a Sport Education unit). Retos, (24), 1620.

DOI: https://doi.org/10.47197/retos.v0i24.34 514
Castillo, G., Corvalán, F., Sazo, N. S., \& Concha, Y. (2017). Efectos de un programa de estimulación físico-cognitiva sobre la autopercepción de la funcionalidad en adultos mayores. Ciencias de la Actividad Física UCM, 18(2), 1-12. DOI: https://doi.org/10.29035/rcaf.18.2.5

Cattuzzo, M. T., Dos Santos Henrique, R., Ré, A. H. N., de Oliveira, I. S., Melo, B. M., de Sousa Moura, M., de Araújo, R. C., \& Stodden, D. (2016). Motor competence and health related physical fitness in youth: A systematic review. Journal of Science and Medicine in Sport, 19(2), 123-129. DOI: 10.1016/j.jsams.2014.12.004

Chaves-Castro, K., Jiménez-Díaz, J., \& Salazar-Rojas, W. (2018). Efectividad de los programas de intervención motriz en el desempeño de los patrones básicos de movimiento: Un meta-análisis. Ágora para la Educación Física y el Deporte, 20(2-3), 182-212. DOI: https://doi.org/10.24197/aefd.23.2018.182-212

Famelia, R., Tsuda, E., Bakhtiar, S., \& Goodway, J. D. (2018). Relationships Among Perceived and Actual Motor Skill Competence and Physical Activity in Indonesian Preschoolers. Journal of Motor Learning and Development, 6(s2), S403-\$423. DOI: 10.1123/jmld.2016-0072

Fu, Y., Gao, Z., Hannon, J., Shultz, B., Newton, M., \& Sibthorp, J. (2013). Influence of a healthrelated physical fitness model on students' physical activity, perceived competence, and enjoyment. Perceptual \& Motor Skills, 777(3), 956-970.

DOI: 10.2466/10.06.PMS.117×32zO

Gabbard, C. P. (2018). Lifelong Motor Development. (7a Ed.). Netherlands: Wolters Kluwer.

Gallahue, D. L., \& Ozmun, J. C. (2005). Understanding Motor Development: Infants, Children, Adolescents, Adults (6a Ed.). Boston: McGraw-Hill Higher Education. 
Jiménez-Díaz, J., Morera-Castro, M., \& Araya-Vargas, G. (2020). Mejorar la competencia motriz no predice un cambio en la autopercepción en adultos. Revista Ciencias de la Actividad Física UCM, №21(2), julio-diciembre, 1-13. DOI: http://doi.org/10.29035/rcaf.21.2.7

Gentil Adarve, M., Zurita Ortega, F., Gómez Sánchez, V., Padial Ruz, R., \& Lara, A. J. (2019). Influencia de la práctica de actividad física en el autoconcepto de adolescentes. Retos, (36),

342-347.

DOI: https://doi.org/10.47197/retos.v36i36.6 8852

Goodway, J. D., Crowe, H., \& Ward, P. (2003). Effects of motor skill instruction of fundamental motor skill development. Adapted Physical Activity Quarterly, 20(3), 298-314. DOI: https://doi.org/10.1123/apaq.20.3.298

Hair, J., Anderson, R., Tatham, R., \& Black, W. (1999). Análisis multivariante. (5.a ed.). Madrid: Prentice Hall.

Haugen, T., Ommundsen, Y., \& Seiler, S. (2013). The relationship between physical activity and physical self-esteem in adolescents: The role of physical fitness indices. Pediatric Exercise Science, 25(1), 138-153. DOI: $10.1123 /$ pes.25.1.138

Holfelder, B., \& Schott, N. (2014). Relationship of fundamental movement skills and physical activity in children and adolescents: A systematic review. Psychology of Sport and Exercise, 382-391. DOI: 10.1016/j.psychsport.2014.03.005

Jiménez, J., Salazar, W., \& Morera, M. (2013). Diseño y validación de un instrumento para la evaluación de patrones básicos de movimiento. Motricidad. European Journal of Human Movement, 31, 87-97. Recuperado de https://www.researchgate.net/publication/ 260084172_DISENO_Y_VALIDACION_DE_ UN_INSTRUMENTO_PARA_LA_EVALUACI ON_DE_PATRONES_BASICOS_DE_MOVIM IENTO
Jiménez-Díaz, J., \& Morera-Castro, M. (2016). Desempeño motor y hábitos de actividad física en estudiantes universitarios en Costa Rica. Movimiento Humano y Salud, 12(2), 15-24. DOI: https://doi.org/10.15359/mhs.122.2

Jiménez-Díaz, J., Morera-Castro, M., \& Araya-Vargas, G. (2018). Validez y confiabilidad del "Perfil de Autopercepción para Adultos" en el ámbito educativo. Sophia Educación, 74(2), 73-83.

DOI: https://doi.org/10.18634/sophiaj.14v.2i.8 28

Jiménez-Díaz, J., Salazar, W., \& Morera-Castro, M. (2015). Age and gender differences in fundamental motor skills. Pensar en Movimiento: Revista de Ciencias del Ejercicio y la Salud, 13(2), 1-16. DOI: 10.15517/pensarmov.v13i2.18327

LeGear, M., Greyling, L., Sloan, E., Bell, R. I., Williams, B.L., Naylor, P.J., \& Temple, V. A. (2012). A window of opportunity? Motor skills and perceptions of competence of children in Kindergarten. International Journal of Behavioral Nutrition and Physical Activity, 9(29), 1-5. Recuperado de https://ijbnpa.biomedcentral.com/articles/1 0.1186/1479-5868-9-29

Lindwall, M., \& Lindgren, E. C. (2005). The effects of a 6-month exercise intervention programme on physical self-perceptions and social physique anxiety in non-physically active adolescent Swedish girls. Psychology of Sport and Exercise, 6(6), 643-658. DOI: https://doi.org/10.1016/j.psychsport.2005.03. 003 
Jiménez-Díaz, J., Morera-Castro, M., \& Araya-Vargas, G. (2020). Mejorar la competencia motriz no predice un cambio en la autopercepción en adultos. Revista Ciencias de la Actividad Física UCM, № 21(2), julio-diciembre, 1-13. DOI: http://doi.org/10.29035/rcaf.21.2.7

Logan, S. W., Robinson, L. E., Wilson, A. E., \& Lucas, W. A. (2012). Getting the fundamentals of movement: A meta-analysis of the effectiveness of motor skill interventions in children. Child: care, health and development, 38(3), 305-315. DOI: https://doi.org/10.1111/j.1365-2214.2011.01307.x

Lubans, D. R., Aguiar, E. J., \& Callister, R. (2010). The effects of free weights and elastic tubing resistance training on physical selfperception in adolescents. Psychology of Sport and Exercise, 77(6), 497-504. DOl: https://doi.org/10.1016/j.psychsport.2010.06. 009

Lubans, D. R., Morgan, P. J., Cliff, D. P., Barnett, L. M., \& Okely, A. D. (2010). Fundamental movement skills in children and adolescents. Sports medicine, 40(12), 10191035. DOI: https://doi.org/10.2165/11536850000000000-00000

Messer, B., \& Harter, S. (2012). The Self-perception profile for adults: Manual and Questionnaires. Denver: Universidad de Denver.

Morano, M., Colella, D., Rutigliano, I., Fiore, P., Pettoello-Mantovani, M., \& Campanozzi, A. (2014). A multi-modal training programme to improve physical activity, physical fitness and perceived physical ability in obese children. Journal of Sports Sciences, 32(4), 345-353. s3h.

DOI: https://doi.org/10.1080/02640414.2013.8246 $\mathrm{O} 2$

Morgan, P. J., Barnett, L. M., Cliff, D. P., Okely, A. D., Scott, H. A., Cohen, K. E., \& Lubans, D. R. (2013). Fundamental Movement Skill Interventions in Youth: A Systematic Review and Meta-analysis. Pediatrics, 132(5), el361-e1383. DOl: https://doi.org/10.1542/peds.2013-1167
Morgan, P. J., Saunders, K., \& Lubans, D. R. (2012). Improving physical self-perception in adolescent boys from disadvantaged schools: Psychological outcomes from the Physical Activity Leaders randomized controlled trial. Pediatric obesity, 7(3), e27e32. DOI: 10.1111/j.2047-6310.2012.00050.x

O'Brien, W., Belton, S., \& Issartel, J. (2016). Fundamental movement skill proficiency amongst adolescent youth. Physical Education and Sport Pedagogy, 27(6), 557571. https://doi.org/10.1080/17408989.2015.10174 51

Opdenacker, J., Delecluse, C., \& Boen, F. (2009). The longitudinal effects of a lifestyle physical activity intervention and a structured exercise intervention on physical selfperceptions and self-esteem in older adults. Journal of sport \& exercise psychology, 37(6), 743-760. DOI: https://doi.org/10.1123/jsep.31.6.743

Piek, J. P., McLaren, S., Kane, R., Jensen, L., Dender, A., Roberts, C., ... Straker, L. (2013). Does the Animal Fun program improve motor performance in children aged 4-6 years? Human Movement Science, 32(5), 10861096.

DOI: https://doi.org/10.1016/j.humov.2012.08.004

Robinson, L. E., Stodden, D. F., Barnett, L. M., Lopes, V. P., Logan, S. W., Rodrigues, L. P., \& D'Hondt, E. (2015). Motor Competence and its Effect on Positive Developmental Trajectories of Health. Sports Medicine, 45(9), 1273-1284. DOI: https://doi.org/10.1007/s40279-015-0351-6

Stein, C., Fisher, L., Berkey, C., \& Colditz, G. (2007). Adolescent physical activity and perceived competence: does change in activity level impact self-perception? Journal of adolescent health, 40(5), 462.el-462.e8. DOI: 10.1016/j.jadohealth.2006.11.147 
Jiménez-Díaz, J., Morera-Castro, M., \& Araya-Vargas, G. (2020). Mejorar la competencia motriz no predice un cambio en la autopercepción en adultos. Revista Ciencias de la Actividad Física UCM, № 21(2), julio-diciembre, 1-13. DOI: http://doi.org/10.29035/rcaf.21.2.7

Stodden, D. F., Goodway, J. D., Langendorfer, S. J., Roberton, M. A., Rudisill, M. E., Garcia, C., \& Garcia, L. E. (2008). A developmental perspective on the role of motor skill competence in physical activity: An emergent relationship. Quest, 60(2), 290306.

DOl: https://doi.org/10.1080/00336297.2008.1048 3582

True, L., Brian, A., Goodway, J., \& Stodden, D. (2017). Relationships Between Product- and Process-Oriented Measures of Motor Competence and Perceived Competence. Journal of Motor Learning and Development, 5(2), 319-335. DOI: https://doi.org/10.1123/jmld.2016-0042

Van Capelle, A., Broderick, C. R., van Doorn, N., Ward, R. E., \& Parmenter, B. J. (2017). Interventions to improve fundamental motor skills in pre-school aged children: A systematic review and meta-analysis. Journal of Science and Medicine in Sport, 20(7), 658666.

DOI:

https://doi.org/10.1016/j.jsams.2016.11.008

Wang, J., Liu, W., \& Bian, W. (2013). Relationship between perceived and actual motor competence among college students. Perceptual and Motor Skills, 176(1), 272-279. DOI:

https://doi.org/10.2466/25.06.PMS.116.1.272279

Wick, K., Leeger-Aschmann, C. S., Monn, N. D., Radtke, T., Ott, L. V., Rebholz, C. E., ... Kriemler, S. (2017). Interventions to Promote Fundamental Movement Skills in Childcare and Kindergarten: A Systematic Review and Meta-Analysis. Sports Medicine, 47(10), 2045-2068. DOI: https://doi.org/10.1007/s40279-017-0723-1

\section{Dirección para correspondencia}

Dra. Judith Jiménez-Díaz

Escuela de Educación Física y Deportes,

Universidad de Costa Rica

San José, Costa Rica

ORCID: https://orcid.org/0000-0001-8663-7413

Contacto:

judith.jimenez_d@ucr.ac.cr

Recibido: 08-01-2020

Aceptado: 18-08-2020 